\title{
Influence of trans-neptunian objects on motion of major planets and limitation on the total TNO mass from planet and spacecraft ranging
}

\author{
E. V. Pitjeva \\ Institute of Applied astronomy RAS, \\ Kutuzov quay 10, 191187 St. Petersburg, Russia \\ email: evp@ipa.nw.ru
}

\begin{abstract}
Perturbations from asteroids and Trans-Neptunian Objects affect significantly on the orbits of planets and should be taken into account when high-accuracy planetary ephemerides are constructed. On the other hand, from an analysis of motion of the major planets by processing of precise measurements of spacecraft a limitation on the total TNO mass may be obtained. To estimate influence of TNO on motion of planets the largest 21 TNO have been included into the process of simultaneous numerical integration, and positions of planets obtained with taking for TNO have been compared with positions of planets of numerical EPM ephemeris of IAA RAS constructed without these objects. The perturbations of other TNO have been modeled by the perturbation from a circular ring having a radius $43 \mathrm{AU}$ and different masses. It has been shown that all the test masses of the TNO ring except the minimum mass $\left(5.26 \cdot 10^{-8} \mathrm{M}_{\odot}\right)$ are too large and make the data residuals worse. Thus, the upper limit of the total mass of all TNO including Pluto, the 21 largest TNO and the TNO ring (with the 43 AU radius) should not exceed $8.04 \cdot 10^{-8} \mathrm{M}_{\odot}$.
\end{abstract}

Keywords. Celestial mechanics, ephemerides, radar astronomy, solar system

\section{Introduction: a precision of observations and dynamical models}

At present, the accuracy of radar observations of spacecraft orbiting near planets reaches a meter precision in distances between the Earth and planets, that is the twelfth figure in distances. The construction of high-precision ephemerides of major planets, which corresponds to meter accuracy of ranging, requires the creation of the adequate mathematical and dynamical model of the motion of planets on the base of General Relativity and that takes into account all perturbating factors.

Perturbations from asteroids and Trans-Neptunian Objects (TNO) affect significantly the orbits of planets and should be taken into account when high-accuracy planetary ephemerides are constructed. On the other hand, from analysis of these perturbations it appears possible to derive values of some physical parameters of the asteroids and TNO, including a limitation on the total TNO mass by processing of precise measurements of spacecraft.

The EPM ephemerides (Ephemerides of planets and the Moon) of IAA RAS originated in the seventies of the last century to support space flights at about the same time as DE ephemerides and have been developed since that time. The EPM2008 ephemerides have been used for data analysis. 


\section{EPM2008 ephemerides}

For the construction of the EPM2008 ephemerides a numerical integration of the equations of motion of the major planets, the Sun, the Moon and the lunar physical libration, asteroids and TNO, taking into consideration perturbations from solar oblateness has been performed in the Parameterized Post-Newtonian metric for the harmonic coordinates $\alpha=0$ and General Relativity values $\beta=\gamma=1$.

A serious problem in the construction of planetary ephemerides arises due to the necessity to take into account the perturbations caused by minor planets. The experiment showed that the fitting of ephemerides accounted for the perturbations from only several biggest asteroids (DE200 and EPM87) to the Viking lander data was poor. The perturbations from 300 and more asteroids have been taken into account in the ephemerides starting with DE403, and EPM98. However, masses of many of these asteroids are quite poorly known, and the accuracy of the planetary ephemerides deteriorates due to this factor.

Masses of most massive asteroids which more strongly affect Mars and the Earth can be estimated from observations of martian landers and spacecraft orbiting Mars. The five of the 300 large asteroids proved to be double and their masses are known now. The masses of Ida(243), Gaspra(951), Eros(433) and Mathilda(253) have been derived by perturbations of the spacecraft during the NEAR flyby. Unfortunately, the classical method of determining masses of asteroids for which close encounters occur can give a accurate determination of asteroid masses only for separate cases when very close encounters are provided with useful data before and after encounters. The masses of the rest of the 301 large asteroids have been estimated by the astrophysical method from analysis of data concerning their diameters and spectral classes estimating the mean densities of the three asteroid taxonomical classes (C, S, M) from ranging observations. However, many of these objects are too small to be observed from the Earth, but their total mass is large enough to affect the orbits of the major planets. The total contribution of all remaining small asteroids is modeled as the acceleration caused by a solid ring with the constant mass distribution in the ecliptic plane (Krasinsky et al., 2002).

At present hundreds of large TNO were revealed including Eris which surpasses Pluto in the mass. The updated model of EPM2008 includes the 21 largest TNO (Eris, Haumea, Makemake, Sedna, Quaoar, Orcus, Varana, Ixion and others) into the process of the simultaneous numerical integration. In order to investigate the influence of trans-neptunian objects on motion of planets, positions of planets obtained with using the two versions of EPM ephemeris with and without the 21 TNO have been compared. The maximum differences obtained in right ascension $(d \alpha)$, declination $(d \delta)$ and heliocentric distance $(d R)$ on the time interval 1913-2020 are shown in Table 1. These differences are small, they are less on the order of the magnitude than the formal uncertainties of planet positions. After adjusting ephemerides to the present set of observational data it wasn't found the difference in residuals for these versions of ephemerides (with and without TNO). However, it turns out that the total shift of the barycenter of the solar system due to the 21 largest trans-Neptunian objects is $6140 \mathrm{~m}$ within the lifetime of GAIA (2011-2020).

Some tests have been made for estimating the effect of other TNO on the motion of planets. Their perturbations have been modeled by the perturbation from a circular ring having a radius of $43 \mathrm{AU}$ and different masses. The minimum mass $\left(\mathrm{EPM}_{1-T N O}\right)$ of this ring is equal to the mass of 100000 bodies with $100 \mathrm{~km}$ in diameter and density is equal to $2 \mathrm{~g} / \mathrm{cm}^{3}$, it amounts to 110 masses of Ceres. The maximum mass $\left(\mathrm{EPM}_{5-T N O}\right)$ of the ring is expected to be 100 times the minimum mass. Masses of $\mathrm{EPM}_{2-T N O}, \mathrm{EPM}_{3-T N O}$, $\mathrm{EPM}_{4-T N O}$ amount $25 \%, 50 \%$, and $75 \%$ of the maximum mass respectively. The effect 
Table 1. The maximum differences in $d \alpha, d \delta$ and $d R, 1913-2020$, for ephemerides with and without the 21 largest TNO.

\begin{tabular}{lccc}
\hline Planet & $d \alpha[$ mas $]$ & $d \delta[$ mas $]$ & $d R[\mathrm{~m}]$ \\
\hline Mercury & 0.006 & 0.003 & 2.4 \\
Venus & 0.006 & 0.010 & 6.1 \\
Mars & 0.068 & 0.025 & 15.5 \\
Jupiter & 0.260 & 0.110 & 199 \\
Saturn & 0.298 & 0.103 & 311 \\
Uranus & 1.688 & 0.744 & 5932 \\
Neptune & 2.321 & 0.279 & 50332 \\
Pluto & 2.578 & 1.064 & 60821 \\
\hline
\end{tabular}

Table 2. The rms residuals in $\mathrm{m}$ and the weight unit errors $\sigma_{0}$ for EPM ephemerides with different masses of the TNO ring.

\begin{tabular}{|c|c|c|c|c|c|c|}
\hline Observations & $\begin{array}{l}\text { Martian } \\
\text { landers }\end{array}$ & $\begin{array}{l}\text { Martian } \\
\text { spacecraft }\end{array}$ & $\begin{array}{c}\text { Venus } \\
\text { Express }\end{array}$ & $\begin{array}{l}\text { Spacecraft } \\
\text { at Jupiter }\end{array}$ & $\begin{array}{l}\text { Spacecraft } \\
\text { at Saturn }\end{array}$ & $\sigma_{0}$ \\
\hline Interval & 1976-1997 & $1998-2008$ & 2006-2007 & $1973-2001$ & 1979-2006 & $1913-2008$ \\
\hline Numbers n.p. & 1348 & 13903 & 547 & 7 & 34 & 97101 \\
\hline EPM2008 & 11.82 & 2.04 & 2.59 & 13.09 & 3.04 & 0.876 \\
\hline $\mathrm{EPM}_{1-T N O}$ & 12.00 & 1.87 & 2.59 & 13.08 & 3.02 & 0.876 \\
\hline $\mathrm{EPM}_{2-T N O}$ & 13.16 & 1.90 & 2.63 & 13.20 & 64.35 & 1.000 \\
\hline $\mathrm{EPM}_{3-T N O}$ & 13.35 & 2.00 & 2.68 & 13.29 & 129.7 & 1.305 \\
\hline $\mathrm{EPM}_{4-T N O}$ & 13.83 & 2.05 & 2.74 & 17.64 & 195.0 & 1.696 \\
\hline $\mathrm{EPM}_{5-T N O}$ & 14.26 & 2.06 & 2.80 & 27.43 & 200.3 & 2.126 \\
\hline
\end{tabular}

of the ring is only noticeable for more accurate observations - the spacecraft data. The rms residuals and the weight unit errors for these data after fitting the standard and test EPM ephemerides are given in Table 2.

It is seen from the above that all the masses of the TNO ring except the minimum mass $\left(\mathrm{EPM}_{1-T N O}=5.26 \cdot 10^{-8} \mathrm{M}_{\odot}\right)$ are too large and make the data residuals worse. These results give a possibility to estimate the upper limit of the total mass of all TNO and to include the mass value of the TNO ring into the set of the adjusted parameters.

Thus, the dynamical model of EPM2008 ephemerides takes into account the following:

- mutual perturbations from major planets, the Sun, the Moon and 5 more massive asteroids;

- perturbations from the other 296 asteroids chosen due to their strong perturbations on Mars and the Earth;

- perturbation from the massive asteroid ring with constant mass distribution in the ecliptic plane;

- perturbations from the 21 largest TNO;

- perturbation from a massive ring of TNO with the radius of $43 \mathrm{AU}$;

- perturbations due to the solar oblateness $J_{2}=2 \cdot 10^{-7}$.

The modern EPM2008 ephemerides have resulted from a least squares adjustment to observational data totaling about 550000 position observations of different types including different American and Russian radiometric observations of planets and spacecraft (VEX, MGS, Odyssey, MRO, Cassini, etc.) 1961-2008, CCD astrometric observations of outer planets and their satellites, meridian transits and photographic observations of the 
Table 3. Masses of Ceres, Pallas, Juno, Vesta, Iris, Bamberga in $10^{-10} M_{\odot}$.

\begin{tabular}{ccccccc}
\hline & Ceres & Pallas & Juno & Vesta & Iris & Bamberga \\
\hline & 4.71 & 1.06 & 0.129 & 1.32 & 0.040 & 0.046 \\
$\sigma_{\text {formal }}$ & \pm 0.007 & \pm 0.003 & \pm 0.003 & \pm 0.001 & \pm 0.001 & \pm 0.001 \\
$\sigma_{\text {real }}$ & \pm 0.03 & \pm 0.03 & \pm 0.008 & \pm 0.03 & \pm 0.008 & \pm 0.008 \\
\hline
\end{tabular}

XX-th century, as well as the VLBI spacecraft data. Data used for the production of ephemerides were taken from databases of the JPL website (http:/ssd.jpl.nasa.gov/iaucomm4/) created by Standish and continuing by Folkner, and extended to include Russian radar observations of planets (//www.ipa.nw.ru/ PAGE/DEPFUND/LEA/ENG/englea. htm). The significance of the high precise radiometric observations of planets beginning in 1961 (and afterward spacecraft) continuing with the increasing accuracy it should be stressed. It has been these observations that have made it possible to determine and improve a broad set of astronomical constants. The detailed discription of the EPM2008 ephemerides are given in the papers by Pitjeva $(2009,2010)$.

\section{Values of the adjusted parameters}

More than 260 parameters have been determined while improving the planetary part of EPM2008. In addition to the orbital elements of all the planets and the main satellites of the outer planets, different physical constants have were estimated including parameters of a surface topography of planets and the rotation of Mars, the ratio masses of the Earth and the Moon, masses of ten asteroids that perturb Mars most strongly, mean densities for three taxonomic classes of asteroids $(\mathrm{C}, \mathrm{S}, \mathrm{M})$, the mass and the radius of the asteroid ring, the mass of the TNO ring.

In Table 3 and further the adjusted value of several of these parameters are presented. The obtained values of parameters are shown with their real uncertainties estimated by comparing the values obtained in dozens of different test LS solutions that differed by the sets of observations, their weights, and the sets of parameters included in the solution, as well as by comparing parameter values produced by independent groups. The discussion of real uncertainties is given in the paper by Pitjeva \& Standish, 2009.

Two parameters that characterize the ring modeling the effect from the rest of small asteroids (its mass $\mathrm{M}_{\text {ring }}$ and radius $\mathrm{R}_{\text {ring }}$ ) have been determined:

$$
M_{\text {ring }}=(0.87 \pm 0.35) \cdot 10^{-10} M_{\odot}, R_{\text {ring }}=(3.13 \pm 0.05) A U .
$$

Thus, the estimation of the total mass of the main belt asteroids represented by the sum masses of 301 asteroids and the asteroid ring is:

$$
M_{\text {belt }}=(13 \pm 2) \cdot 10^{-10} M_{\odot}\left(\text { about } 3 M_{\text {Ceres }}\right) .
$$

The mass value of the ring of TNO has been obtained:

$$
M_{\text {TNOring }}=(498 \pm 14) \cdot 10^{-10} M_{\odot}(5 \sigma)
$$


Thus, the total mass of all TNO including Pluto, the 21 largest TNO and the TNO ring of other TNO objects with the $43 \mathrm{AU}$ radius is:

$$
M_{T N O}=775 \cdot 10^{-10} M_{\odot}\left(\text { about } 164 M_{\text {Ceres }} \text { or } 2 M_{M o o n}\right)
$$

\section{References}

Krasinsky, G. A., Pitjeva, E. V., Vasilyev, M. V., \& Yagudina, E. I. 2002, Icarus, 158, 98

Pitjeva, E. V. 2009, in: M. Soffel \& N. Capitane (eds.), Astrometry, Geodynamics and Astronomical Reference Systems, Proc. JOURNEES-2008 (Dresden), p. 57

Pitjeva, E. V. \& Standish, E. M. 2009, Celest. Mech. Dyn. Astr., 103, 365

Pitjeva, E. V. 2010, in: S. Klioner, P. K. Seidelmann \& M. Soffel (eds.), Relativity in fundamental astronomy, IAUS 261, (Cambridge: Cambridge University Press), in print 